\title{
The distribution of inverses modulo a prime in short intervals
}

by

\author{
S. M. Gonek, G. S. Krishnaswami and \\ V. L. SondHI (Rochester, NY)
}

Let $\bar{\nu}$ denote the multiplicative inverse of $\nu$ modulo an odd prime $p$ and set

$$
\mathcal{N}=\{\bar{\nu}(\bmod p): M<\nu \leq M+N\},
$$

where $M \geq 0$ and $N \geq 1$ are integers such that $(M, M+N] \subseteq(0, p)$. The elements of $\mathcal{N}$ are known to be well-distributed in various senses. For instance, C. Cobeli [1] has shown that the fractional parts of representatives of $\mathcal{N}$ divided by $p$ are uniformly distributed $(\bmod 1)$ when $N \gg p^{1 / 2+\varepsilon}$.

Here we wish to study the distribution of the elements of $\mathcal{N}$ in "short" intervals $(m, m+H], 1 \leq m \leq p$, where $H<p$. To this end we set

$$
f(m, H)=|\{n \in(m, m+H]: n(\bmod p) \in \mathcal{N}\}|
$$

(here || denotes cardinality) and estimate

$$
\mathcal{M}_{k}(H, p)=\sum_{m=1}^{p}(f(m, H)-H N / p)^{k} .
$$

Since each element of $\mathcal{N}$ is counted in exactly $H$ of the intervals $(m, m+H]$, $1 \leq m \leq p$, the mean of $f(m, H)$ is

$$
\frac{1}{p} \sum_{m=1}^{p} f(m, H)=H N / p .
$$

Therefore, $\mathcal{M}_{k}(H, p)$ is the $k$ th moment of $f(m, H)$ about its mean. Now the probability that an integer selected at random from $[1, p]$ is congruent to an element of $\mathcal{N}$ is $N / p$. Thus, if the "events" $m+h(\bmod p) \in \mathcal{N}, 1 \leq h \leq H$, were independent, we should have

$$
\mathcal{M}_{k}(H, p)=\mu_{k}(H, N / p) p,
$$

2000 Mathematics Subject Classification: Primary 11N69; Secondary 11K31, 11K45, $11 \mathrm{~T} 23$

Research of the first author was supported in part by grants from the NSF and NSA. 
where $\mu_{k}(H, P)$ is the $k$ th moment of a binomial random variable $X$ with parameters $H$ and $P$. That is,

$$
\mu_{k}(H, P):=E\left((X-H P)^{k}\right)=\sum_{h=1}^{H}\left(\begin{array}{c}
H \\
h
\end{array}\right) P^{h}(1-P)^{H-h}(h-H P)^{k} .
$$

We note that $\mu_{1}(H, P)=0$ and $\mu_{2}(H, P)=H P(1-P)$. C. Cobeli [1] has recently shown that

$$
\mathcal{M}_{2}(H, p)=\mu_{2}(H, N / p) p+O\left(H^{2} p^{1 / 2} \log ^{2} p\right) .
$$

Our main result extends this to larger values of $k$. Then

Theorem. Let $k, N$ and $H$ be positive integers with $1 \leq N, H<p$.

$$
\begin{aligned}
\mathcal{M}_{k}(H, p) & =\sum_{m=1}^{p}(f(m, H)-N H / p)^{k} \\
& =\mu_{k}(H, N / p) p+O\left(H^{k} p^{1 / 2} \log ^{k} p\right) .
\end{aligned}
$$

Here and elsewhere, unless otherwise indicated, implied constants depend on $k$.

One can show (see Montgomery and Vaughan [3]) that for a fixed $k$,

$$
\mu_{k}(H, P) \ll(H P)^{[k / 2]}+H P
$$

uniformly for $0 \leq P \leq 1$ and $H=1,2, \ldots$ Thus our theorem immediately leads to an upper bound for $\mathcal{M}_{k}(H, p)$. Then

Corollary 1. Let $k, H$ and $N$ be positive integers with $1 \leq H, N<p$.

$$
\mathcal{M}_{k}(H, p) \ll p(H N / p)^{[k / 2]}+H N+H^{k} p^{1 / 2} \log ^{k} p .
$$

One can also show (see [3]) that

$$
\mu_{k}=\left(\nu_{k}+o(1)\right)(H P(1-P))^{k / 2}
$$

as $H P(1-P) \rightarrow \infty$, where

$$
\nu_{k}= \begin{cases}1 \cdot 3 \cdot \ldots \cdot(k-1) & \text { if } k \text { is even, } \\ 0 & \text { if } k \text { is odd }\end{cases}
$$

denotes the moments of a normal random variable with mean 0 and standard deviation 1. Using this together with our theorem, we obtain then

Corollary 2. If $H=o\left(p^{1 /(2 k)} / \log p\right)$ and $(H N / p)(1-N / p) \rightarrow \infty$,

$$
\mathcal{M}_{k}(H, p)=\left(\nu_{k}+o(1)\right) p((H N / p)(1-N / p))^{k / 2} .
$$

Thus, $f(m, H)$ is approximately normal with mean $N H / p$ and variance $(H N / p)(1-N / p)$ in appropriate ranges of $H$ and $N$. 
Our final result is an estimate for the moments of gaps between consecutive terms of $\mathcal{N}$. Let $n_{1}, \ldots, n_{N}$ be representatives of the residue classes comprising $\mathcal{N}$ lying in $(0, p)$ and arranged in increasing order. Also set

$$
S_{\kappa}(p)=\sum_{i=1}^{N-1}\left(n_{i+1}-n_{i}\right)^{\kappa} .
$$

From Corollary 1 we shall deduce

Corollary 3. Let $\varepsilon$ be an arbitrarily small positive number and let $\kappa$ be any positive number less than $3 / 2$. Then

$$
S_{\kappa}(p) \ll N(N / p)^{-\kappa}
$$

for $1 \leq N<p$ when $0<\kappa \leq 1$, and for $p^{3 /(2(3-\kappa))+\varepsilon} \ll N<p$ when $1<\kappa<3 / 2$. We also have

$$
S_{\kappa}(p) \gg N(N / p)^{-\kappa}
$$

for $p^{3 / 4+\varepsilon} \ll N<p$ and all $0<\kappa<3 / 2$. In particular, for $0<\kappa<3 / 2$ we have

$$
S_{\kappa}(p) \approx N(N / p)^{-\kappa},
$$

provided that $p^{\max \{3 / 4,3 /(2(3-\kappa))\}+\varepsilon} \ll N<p$.

1. Proof of the Theorem. For the convenience of the reader we state two necessary lemmas without proof. The first, a special case of Theorem 1 in [2], depends on the Riemann hypothesis for curves.

Lemma 1. Let $p, N$, and $\mathcal{N}$, be as above and let $a_{1}, \ldots, a_{s}$ be distinct integers $(\bmod p)$ with $s \leq N$. Then

$$
\sum_{\substack{1 \leq x \leq p \\ x+a_{i}(\bmod p) \in \mathcal{N} \\(1 \leq i \leq s)}} 1=p(N / p)^{s}+O\left(s p^{1 / 2} \log ^{s} p\right)
$$

uniformly for $1 \leq s \leq N<p$. Here the constant implied by the $O$-term is absolute.

A proof of our second lemma may be found in Montgomery and Vaughan [3].

Lemma 2. Let $\mu_{k}(H, P)$ be as in the Theorem. Then

$$
\mu_{k}(H, P)=\sum_{r=0}^{k}\left(\begin{array}{l}
k \\
r
\end{array}\right)(-H P)^{k-r}\left(\sum_{t=0}^{r}\left(\begin{array}{c}
H \\
t
\end{array}\right) S(r, t) t ! P^{t}\right),
$$

where $S(r, t)$ denotes a Stirling number of the second kind, that is, the number of partitions of a set of cardinality $r$ into exactly $t$ non-empty subsets. 
We now proceed with the proof of the Theorem. Expanding the righthand side of (1) by the binomial theorem and taking the sum over $m$ inside, we find that

$$
\mathcal{M}_{k}(H, p)=\sum_{r=0}^{k}\left(\begin{array}{l}
k \\
r
\end{array}\right)(-H N / p)^{k-r} \sum_{m=1}^{p} f(m, H)^{r} .
$$

Here we use the convention that $f(m, H)^{0}=1$ even when $f(m, H)=0$. Let us set

$$
M_{r}(H)=\sum_{m=1}^{p} f(m, H)^{r} .
$$

Then we have $M_{0}(H)=p$, and for $r \geq 1$,

$$
M_{r}(H)=\sum_{\substack{x_{1}=1 \\ x_{1}(\bmod p) \in \mathcal{N}}}^{p} \cdots \sum_{\substack{x_{r}=1 \\ x_{r}(\bmod p) \in \mathcal{N}}}^{p} \sum_{\substack{m=1 \\ m \leq x_{i} \leq m+H \\(1 \leq i \leq r)}}^{p} 1 .
$$

Let $\mathcal{B}$ be a subset of $t \leq r$ distinct elements of $[1, p)$, each of which is congruent $(\bmod p)$ to some element of $\mathcal{N}$. By the definition of $S(r, t)$, the Stirling number of the second kind, we see that the number of maps from a set of cardinality $r$ onto a set of cardinality $t$ is $S(r, t) t$ !. Hence, this is also the number of terms in the $r$ outer sums on the right-hand side of (3) for which $\left\{x_{1}, \ldots, x_{r}\right\}=\mathcal{B}$. We therefore obtain

$$
M_{r}(H)=\sum_{t=1}^{r} S(r, t) t ! \sum_{\substack{\mathcal{B}(\bmod p) \subseteq \mathcal{N} \\|\mathcal{B}|=t}} \sum_{\substack{m \subseteq(m, m+H] \\ \mathcal{B} \subseteq(m, m}}^{p} 1 .
$$

Here $\mathcal{B}(\bmod p) \subseteq \mathcal{N}$ means that $x(\bmod p) \in \mathcal{N}$ for each $x \in \mathcal{B}$. Writing

$$
d(\mathcal{B})=\max _{x_{i}, x_{j} \in \mathcal{B}}\left|x_{i}-x_{j}\right|,
$$

we see that the innermost sum equals $\max (0, H-d(\mathcal{B}))$. Thus, grouping terms according to the size of $d(\mathcal{B})$ as well as $t$, we find that

$$
\begin{aligned}
M_{r}(H) & =\sum_{t=1}^{r} S(r, t) t ! \sum_{d=0}^{H-1}(H-d) \sum_{\substack{\mathcal{B} \subseteq \mathcal{N} \\
|\mathcal{B}|=t \\
d(\mathcal{B})=d}} 1 \\
& =\sum_{t=1}^{r} S(r, t) t ! \sum_{d=0}^{H-1}(H-d) N(t, d),
\end{aligned}
$$

say. Note that $N(1,0)=N$, while $N(1, d)=0$ for $d>0$. For $t>1$, if we set 
$a_{1}=0$ and $a_{t}=d$, then we find that

$$
N(t, d)=\sum_{\substack{1 \leq a_{2}, \ldots, a_{t-1}<d \\ a_{i} \text { distinct }}} \sum_{\substack{1 \leq x \leq p \\ x+a_{i}(\bmod p) \in \mathcal{N} \\(1 \leq i \leq t)}} 1 .
$$

The inner sum equals $p(N / p)^{t}+O\left(t p^{1 / 2} \log ^{t} p\right)$ by Lemma 1 , and this is counted $\left(\begin{array}{l}d-1 \\ t-2\end{array}\right)$ times by the outer sum. Hence, for $t>1$,

$$
N(t, d)=p\left(\begin{array}{l}
d-1 \\
t-2
\end{array}\right)(N / p)^{t}+O\left(d^{t-2} p^{1 / 2} \log ^{t} p\right) .
$$

Note that the implicit constant in the $O$-term depends on $t$, so ultimately on $k$, but not on $p$ or $d$. Using these estimates in (4), we obtain

$$
\begin{aligned}
M_{r}(H)= & H N+\sum_{t=2}^{r} S(r, t) t ! \\
& \times \sum_{d=0}^{H-1}(H-d)\left(p\left(\begin{array}{l}
d-1 \\
t-2
\end{array}\right)(N / p)^{t}+O\left(d^{t-2} p^{1 / 2} \log ^{t} p\right)\right) \\
= & H N+p \sum_{t=2}^{r} S(r, t) t !(N / p)^{t} \sum_{d=0}^{H-1}(H-d)\left(\begin{array}{l}
d-1 \\
t-2
\end{array}\right) \\
& +O\left(H^{r} p^{1 / 2} \log ^{r} p\right)
\end{aligned}
$$

for $r \geq 1$. Here it is to be understood that if $r=1$ the sum vanishes.

The sum over $d$ may be evaluated using the relation $\left(\begin{array}{l}i \\ j\end{array}\right)=\frac{i}{j}\left(\begin{array}{l}i-1 \\ j-1\end{array}\right)$ and the identity

$$
\left(\begin{array}{l}
0 \\
j
\end{array}\right)+\left(\begin{array}{l}
1 \\
j
\end{array}\right)+\ldots+\left(\begin{array}{l}
l \\
j
\end{array}\right)=\left(\begin{array}{l}
l+1 \\
j+1
\end{array}\right)
$$

From these we find that

$$
\sum_{d=0}^{H}(H-d)\left(\begin{array}{c}
d-1 \\
t-1
\end{array}\right)=\left(\begin{array}{c}
H \\
t
\end{array}\right)
$$

so that

$$
M_{r}(H)=H N+p \sum_{t=2}^{r} S(r, t) t !(N / p)^{t}\left(\begin{array}{c}
H \\
t
\end{array}\right)+O\left(H^{r} p^{1 / 2} \log ^{r} p\right) .
$$

As $S(r, 1)=1$ for $r \geq 1$, we can include the term $H N$ in the sum by beginning it at $t=1$. Moreover, since $S(r, 0)=0$ for $r \geq 1$, we may add the term $t=0$ in as well. Thus, we find that when $r \geq 1$,

$$
M_{r}(H)=p \sum_{t=0}^{r} S(r, t) t !(N / p)^{t}\left(\begin{array}{c}
H \\
t
\end{array}\right)+O\left(H^{r} p^{1 / 2} \log ^{r} p\right) .
$$


Finally, using the convention $S(0,0)=1$ and recalling our initial observation that $M_{0}(H)=p$, we see that (5) actually holds for $r \geq 0$.

Using this in (2) and then applying Lemma 2, we obtain

$$
\begin{aligned}
\mathcal{M}_{k}(H, p)= & p \sum_{r=0}^{k}\left(\begin{array}{c}
k \\
r
\end{array}\right)(-H N / p)^{k-r} \sum_{t=0}^{r}\left(\begin{array}{c}
H \\
t
\end{array}\right) S(r, t) t !(N / p)^{t} \\
& +O\left(H^{k} p^{1 / 2} \log ^{k} p\right) \\
= & p \mu_{k}(H, N / p)+O\left(H^{k} p^{1 / 2} \log ^{k} p\right) .
\end{aligned}
$$

This completes the proof of the Theorem.

2. Proof of Corollary 3. To prove Corollary 3 we modify an argument of Montgomery and Vaughan [3]. Set

$$
D(x)=\sum_{\substack{i=1 \\ n_{i+1}-n_{i}>x}}^{N-1} 1 .
$$

Then we have

$$
S_{\kappa}(p)=\kappa \int_{0}^{p} D(x) x^{\kappa-1} d x .
$$

We first establish the upper bound. For $0 \leq x \leq 4 p / N$ we use the trivial estimate $D(x) \leq N$ and find that

$$
\kappa \int_{0}^{4 p / N} D(x) x^{\kappa-1} d x \leq N(4 p / N)^{\kappa} \ll N\left(\frac{N}{p}\right)^{-\kappa} .
$$

We bound $D(x)$ for larger $x$ by noting that if $n_{i+1}-n_{i}>H$, then

$$
\sum_{\substack{m<n<m+H \\ n(\bmod p) \in \mathcal{N}}} 1-H N / p=-H N / p
$$

for $n_{i} \leq m<n_{i+1}-H$. Thus, if $k$ is a non-negative integer, we have

$$
\sum_{\substack{i=1 \\ n_{i+1}-n_{i}>H}}^{N-1}\left(n_{i+1}-n_{i}-H\right)(H N / p)^{2 k} \leq \mathcal{M}_{2 k}(H, p) .
$$

Now suppose that $H N \geq p$. Then by Corollary 1 the right-hand side of (8) is

$$
\ll p(H N / p)^{k}+H^{2 k} p^{1 / 2} \log ^{2 k} p .
$$

Moreover, by the definition of $\mathcal{M}_{k}(H, p)$ this also holds when $k=0$. On the 
other hand, taking $H=[x / 2]$, we see that the left-hand side of $(8)$ is

$$
\geq \sum_{\substack{i=1 \\ n_{i+1}-n_{i}>x}}^{N-1}\left(n_{i+1}-n_{i}-H\right)(H N / p)^{2 k} \geq H(H N / p)^{2 k} D(x) .
$$

Thus, for $x \geq 4 p / N$ we find that

$$
D(x) \ll N(x N / p)^{-k-1}+(N / p)^{-2 k} x^{-1} p^{1 / 2} \log ^{2 k} p .
$$

Suppose first that $0<\kappa<1$. Taking $k=0$ in the above, we obtain

$$
\int_{4 p / N}^{p} D(x) x^{\kappa-1} d x \ll p \int_{4 p / N}^{p} x^{\kappa-2} d x \ll N(N / p)^{-\kappa},
$$

for $1 \leq N<p$. On the other hand, if $\kappa>1$, we choose $k$ large enough so that $k+1>\kappa$ (so, in particular, $k \geq 1$ ), and obtain

$$
\begin{aligned}
\int_{4 p / N}^{p} D(x) x^{\kappa-1} d x \ll & N(N / p)^{-k-1} \int_{4 p / N}^{p} x^{\kappa-k-2} d x \\
& +(N / p)^{-2 k} p^{1 / 2} \log ^{2 k} p \int_{4 p / N}^{p} x^{\kappa-2} d x \\
\ll & N(N / p)^{-\kappa}\left(1+(N / p)^{\kappa-2 k-1} p^{\kappa-3 / 2} \log ^{2 k} p\right) .
\end{aligned}
$$

Hence, we deduce in this case also that

$$
\int_{4 p / N}^{p} D(x) x^{\kappa-1} d x \ll N(N / p)^{-\kappa}
$$

provided that

$$
p^{\frac{2 k-1 / 2}{2 k-\kappa+1}} \log ^{\frac{2 k}{2 k-\kappa+1}} p \leq N<p \quad \text { and } \quad k+1>\kappa .
$$

Note that in order for the $N$-range to be non-trivial when $k \geq 1$, we must have $\kappa<3 / 2$. Thus, upon combining (6), (7), (9) and (10), we find

$$
S_{\kappa}(p) \ll N(N / p)^{-\kappa}
$$

for $1 \leq N<p$ if $0<\kappa<1$, and for $p^{\frac{2 k-1 / 2}{2 k-\kappa+1}} \log ^{\frac{2 k}{2 k-\kappa+1}} p \leq N<p$ if $1<\kappa<3 / 2$, where $k$ is any integer such that $k+1>\kappa$. When $1<\kappa<3 / 2$, we achieve the largest $N$-range by minimizing the exponent

$$
\frac{2 k-1 / 2}{2 k-\kappa+1}=1-\frac{3 / 2-\kappa}{2 k-\kappa+1}
$$

of $p$ subject to $k+1>\kappa$. The minimum clearly occurs when $k=1$, so we obtain (11) for $p^{3 /(2(3-\kappa))} \log ^{2 /(3-\kappa)} p \leq N<p$. Finally, we note that when $\kappa=1$, (11) follows from the definition of $S_{1}(p)$ for any $N$ such that $1 \leq N<p$. This gives the upper bound stated in Corollary 3 . 
To treat the lower bound we again consider the cases $0<\kappa<1$ and $\kappa \geq 1$ separately. First suppose that $\kappa \geq 1$. By Hölder's inequality we have

$$
S_{1}(p)^{\kappa} \leq N^{\kappa-1} S_{\kappa}(p)
$$

and we require a lower bound for $S_{1}(p)=n_{N}-n_{1}$. By Lemma 1 with $s=2, a_{1}=0$, and $a_{2}=(p-1) / 2$, say, it follows that there is a pair of elements of $\mathcal{N}$ that are $\gg p$ apart, provided that $N \gg p^{3 / 4} \log p$. Hence $S_{1}(p) \gg p$ for such $N$, and we deduce from (11) that

$$
S_{\kappa}(p) \gg N\left(\frac{N}{p}\right)^{-\kappa}
$$

For $0<\kappa<1$ we apply Hölder's inequality in the form

$$
S_{1}(p)^{q} \leq S_{\kappa}(p)\left(S_{(q-\kappa) /(q-1)}(p)\right)^{q-1},
$$

where $q$ is any real number greater than 1 . We have $S_{1}(p) \gg p$ when $N \gg$ $p^{3 / 4} \log p$, as before, and also the upper bound

$$
S_{(q-\kappa) /(q-1)}(p) \ll N(N / p)^{-(q-\kappa) /(q-1)}
$$

for $1<(q-\kappa) /(q-1)<3 / 2$ and $p^{\frac{3}{2}\left(3-\frac{q-\kappa}{q-1}\right)+\varepsilon / 2} \ll N<p$. It therefore follows, on taking $q$ sufficiently large, that

$$
S_{\kappa}(p) \gg p^{q} /\left(N^{q-1}(N / p)^{\kappa-q}\right)=N(N / p)^{-\kappa}
$$

for $p^{3 / 4+\varepsilon} \ll N<p$. This gives the required lower bound.

The final assertion of the corollary follows immediately on combining the upper and lower bounds for $S_{\kappa}(p)$.

\section{References}

[1] C. I. Cobeli, Topics in the distribution of inverses $(\bmod q)$, doctoral dissertation, University of Rochester, 1997.

[2] C. I. Cobeli, S. M. Gonek and A. Zaharescu, The distribution of inverses modulo a prime, to appear.

[3] H. L. Montgomery and R. C. Vaughan, On the distribution of reduced residues, Ann. of Math. 123 (1986), 311-333.

Department of Mathematics

University of Rochester

Rochester, NY 14627, U.S.A.

E-mail: gonek@math.rohester.edu 